\title{
A NOTE ON THE PRINCIPLE OF MININUM POTENTIAL ENERGY FOR LINEAR ANISOTROPIC ELASTIC SOLIDS*
}

\author{
By M. E. GURTIN (Brown University)
}

Introduction. The displacement equations of equilibrium in the linear theory of homogeneous elastic solids, with reference to rectangular cartesian coordinates $x_{i}$ and in the usual indicial notation $\dagger$, appear as

$$
c_{i j k l} u_{k, l i}+f_{i}=0,
$$

where

$$
\begin{aligned}
c_{i j k l} & =c_{k l i j}, \\
c_{i j k l} & =c_{i i k l} .
\end{aligned}
$$

Here $u_{i}(\mathbf{x})$ and $f_{i}(\mathbf{x})$ are the cartesian components of the displacement vector $\mathbf{u}(\mathbf{x})$ and of the body-force density $\mathbf{f}(\mathbf{x})$, respectively, while $c_{i j k l}$ are the cartesian components of the constant elasticity tensor $\mathbf{c}$. The present paper is concerned exclusively with the displacement boundary-value problem of elastostatics. This problem consists in determining a vector field $\mathbf{u}(\mathbf{x})$ which meets (1) throughout the region of space $D$ occupied by the medium, subject to the boundary condition

$$
\mathbf{u}=\mathbf{u}^{*}(\mathbf{x}) \text { on } B
$$

where $B$ is the boundary of $D$.

The principle of minimum potential energy (as applied to the displacement boundaryvalue problem) asserts that among the vector fields satisfying the boundary condition (4) 'one which also meets the field equations (1) on $D$ is characterized by an absolute minimum of the functional

$$
\Phi[u]=\frac{1}{2} \int_{D} c_{i j k l} u_{i, i} u_{k, l} d V-\int_{D} f_{i} u_{i} d V .
$$

The conventional proof of the principle rests on the assumption that the elastic constants satisfy the symmetry conditions (2), (3), as well as inequalities which are necessary and sufficient for the positive definiteness of the strain-energy density. Thus it is assumed that

$$
c_{i j k l} e_{i j} e_{k l}>0 \text { if } \mathrm{e} \neq 0,
$$

for every symmetric second-order tensor e.

The question as to whether the foregoing requirements on c are necessary for the validity of the minimum principle is of theoretical interest and is relevent to certain

*Received February 9, 1962. Revised manuscript received March 14, 1962. The results communicated in this paper were obtained in the course of an investigation conducted under Contract Nonr562(25) of Brown University with the Office of Naval Research in Washington, D. C.

†Throughout this paper Latin subscripts range over the integers $(1,2,3)$ and summation over repeated subscripts is implied. Subscripts preceded by a comma indicate differentiation with respect to the corresponding cartesian coordinate. Letters in boldface designate tensors. 
considerations in nonlinear elasticity theory. It is the purpose of the present note to show that the principle continues to hold for the displacement boundary-value problem if the tensor c meets merely (2), as well as

$$
c_{i j k l} \xi_{i} \xi_{k} \eta_{i} \eta_{l}>0 \text { if } \xi \neq 0, \quad n \neq 0,
$$

for every pair of vectors $\xi$ and $\boldsymbol{n}$. The inequality (7) represents a necessary and sufficient condition that the system of partial differential equations (1) be strongly elliptic [1]. Toupin and Bernstein [2] have shown that (7) admits a simple intrinsic interpretation: an (anisotropic) elastic body propagates plane waves with positive real speeds if and only if (7) holds. Finally we note that (2), (3), and (6) together imply (7).

A generalization of the principle of minimum potential energy. In what follows we assume that the region occupied by the solid is a bounded, regular region of space, i.e. a closed region $D+B$ whose boundary $B$ consists of a finite number of non-intersecting closed regular surfaces, the latter term being used in the sense of Kellogg [3].

TheOREM. Let $\mathbf{u}(\mathbf{x})$ be twice continuously differentiable on $D+B$ and meet the partial differential equation (1) on $D$, as well as the boundary condition (4) on $B$. Let $\mathbf{c}$ satisfy the symmetry condition (2) and the strong ellipticity condition (7). Let $K$ be the class of (kinematically admissible) vector fields $\mathbf{u}^{\mathbf{0}}(\mathbf{x})$ which are twice continuously differentiable on on $D+B$ and meet

$$
\mathfrak{u}^{0}=\mathbf{u}^{*}(\mathbf{x}) \text { on } \quad B
$$

Then

$$
\Phi[\mathbf{u}]=\min \Phi\left[\mathbf{u}^{0}\right] \text { over all } \mathbf{u}^{0} \varepsilon K
$$

where $\Phi$ is the functional defined by (5), and this absolute minimum is assumed by $\Phi\left[\mathbf{u}^{0}\right]$ only if $\mathfrak{u}=\mathbf{u}^{0}$ on $D+B$.

With a view toward a proof of this theorem we cite the following far reaching lemma due to van Hove [4].

Lemma. Let $\mathbf{\nabla}(\mathbf{x})$ be twice continuously differentiable on $D+B$, with $\mathbf{\nabla}=0$ on $B$. Let $\mathbf{c}$ be a fourth order tensor which meets (7). Then

$$
\int_{D} c_{i j k l} v_{i, j} v_{k, l}>0,
$$

if $\nabla$ does not vanish identically on $D+B$.

We now turn to the proof of our theorem. First, it is evident that $\mathfrak{u} \varepsilon K$. Thus it is sufficient to show that

$$
\Phi[\mathbf{u}]<\Phi\left[\mathbf{u}^{0}\right],
$$

if $\mathfrak{u}$ is not identically equal to $\mathfrak{u}^{0}$ on $D$. To this end let

$$
\mathbf{u}^{\prime}=\mathbf{u}^{0}-\mathbf{u} \text { on } D .
$$

Then, by (2),

$$
c_{i j k l} u_{i, j}^{0} u_{k, l}^{0}=c_{i j k l} u_{i, j}^{\prime} u_{k, l}^{\prime}+c_{i j k l} u_{i, j} u_{k, l}+2 c_{i j k l} u_{i, j}^{\prime} u_{k, l},
$$

and hence, using (5) and (12),

$$
\Phi\left[\mathbf{u}^{0}\right]-\Phi[\mathbf{u}]=\frac{1}{2} \int_{D} c_{i j k l} u_{i, j}^{\prime} u_{k, l}^{\prime} d V+\int_{D} c_{i j k l} u_{i, j}^{\prime} u_{k, l} d V-\int_{D} f_{i} u_{i}^{\prime} d V .
$$


In view of (4), (8), and (12),

$$
\mathbf{u}^{\prime}=0 \text { on } B
$$

Thus, from (15), (1), and the divergence theorem

$$
\int_{D} c_{i j k l} u_{i, j}^{\prime} u_{k, l} d V=-\int_{D} c_{i j k l} u_{k, l} u_{i}^{\prime} d V=\int_{D} f_{i} u_{i}^{\prime} d V
$$

The inequality (11) now follows from (14), (16), and van Hove's lemma. This completes the proof.

Discussion. The uniqueness of the solution to the boundary-value problem characterized by (1) and (4) is a corollary of the preceding theorem*, if c meets (2) and (7).

For an isotropic elastic solid we have in particular

$$
c_{i j k l}=\mu\left[\delta_{i k} \delta_{j l}+\delta_{i l} \delta_{i k}+\frac{2 \sigma}{1-2 \sigma} \delta_{i j} \delta_{k l}\right],
$$

while (6) becomes

$$
\mu>0, \quad-1<\sigma<\frac{1}{2}
$$

where $\mu$ and $\sigma$, respectively, denote the shear modulus and Poisson's ratio of the material. That (18) is not necessary for the truth of the principle of minimum potential energy was apparently observed first by Hill [6], who showed that the principle, with limitation to the displacement boundary-value problem, holds if (18) is replaced by the less restrictive assumption**

$$
\mu>0, \quad-\infty<\sigma<\frac{1}{2}, \quad 1<\sigma<\infty .
$$

But (19), by virtue of (17), is equivalent to (7). Thus the theorem given in the present paper is a generalization to anisotropic elastic solids of Hill's result for the isotropic case. When $\mu<0$ the minimum energy principle gives way to an analogous maximum principle. Finally, it is clear from counter-examples due to Ericksen and Toupin [7] and Ericksen [8] that uniqueness of the solution to the displacement boundary-value problem fails when $\mu=0$ or $\frac{1}{2}<\sigma \leq 1$. Since the minimum principle implies the uniqueness theorem, (19), and hence (7), cannot be further weakened. $\dagger$

Acknowledgement. This note is the result of correspondence with Dr. R. A. Toupin, who directed the author's attention to van Hove's lemma. The author is grateful to Professor E. Sternberg for his helpful criticisms of the manuscript.

\section{REFERENCES}

1. C. B. Morrey, Jr., Second order elliptic systems of differential equations, Annals Math. Stud. 33, 101 (1954)

2. R. A. Toupin and B. Bernstein, Sound waves in deformed perfectly elastic materials. Acousto-elastic effect, J. Acoust. Soc. 33, 216 (1961)

3. O. D. Kellogg, Foundations of potential theory, Springer, Berlin, 1929

*This corollary may be established in the same manner as the analogous corollary for the isotropic elastic solid [5]. Dr. R. A. Toupin, in a private communication, has furnished the author with a direct proof of this uniqueness theorem based on van Hove's lemma. In fact, Toupin's argument is valid even if (2) fails to hold. In this connection see also Morrey [1].

**The same conclusion was reached independently by different means in [5].

$\dagger$ Note that $\sigma \neq 1 / 2$ is implicit in (17); the case of the incompressible medium requires a separate treatment. 
4. L. van Hove, Sur l'extension de la condition de Legendre du calcul de variations aux intégrales multiples à plusieurs fonctions inconnues, Konink. Nederl. Akad. Wetens. 50, 18 (1947)

5. M. E. Gurtin and Eli Sternberg, On the first boundary-value problem of linear elastostatics, Arch. Rat. Mech. Anal. 6, 177 (1960)

6. R. Hill, On uniqueness and stability in the theory of finite elastic strain, J. Mech. Phys. Solids 5, 229 (1957)

7. J. L. Ericksen and R. A. Toupin, Implications of Hadamard's condition for elastic stability with respect to uniqueness theorems, Canad. J. Math. 8, 432 (1956)

8. J. L. Ericksen, On the Dirichlet problem for linear differential equations, Proc. Amer. Math. Soc. 8, 521 (1957) 